\title{
Quantifying Numerical Errors in the Simulation of Transcranial Ultrasound using Pseudospectral Methods
}

\author{
James L. Robertson*, Ben T. Cox, and Bradley E. Treeby \\ Department of Medical Physics and Biomedical Engineering, University College London, UK \\ *Email: james.robertson.10@ucl.ac.uk
}

\begin{abstract}
Effective transcranial transmission of focused ultrasound is desirable for various therapeutic applications. Time-reversal (TR) focusing based on numerical simulations of ultrasound propagation can be used to correct for the aberrating skull layer. For weakly heterogeneous media, k-space and pseudospectral time domain (PSTD) methods have been shown to have increased accuracy and efficiency compared to the finitedifference time domain (FDTD) methods typically used in TR. However, their suitability for highly heterogeneous, transcranial simulations is less clear. Here, this is established in terms of spatial and temporal sampling requirements through numerical testing and comparison with FDTD schemes. PSTD schemes are shown to give equal or better accuracy compared to FDTD schemes for modelling propagation through tissue-realistic heterogeneities, which, combined with the reduction in numerical dispersion obtained with k-space correction, recommends them for use in simulated TR.
\end{abstract}

Index Terms - Error measurement, transcranial focusing, heterogeneous media, k-space correction, pseudospectral methods

\section{INTRODUCTION}

Recently there has been renewed interest in the focused transmission of ultrasound across the skull for the purposes of adapting high intensity focused ultrasound (HIFU) for use in the brain, ultrasonic disruption of the blood brain barrier for targeted drug delivery, and the novel technique of ultrasonic modulation and stimulation of neural tissue, which was recently carried out in humans for the first time [1, 2].

The primary obstacle to focusing ultrasound into the brain is the skull. Bone has a high acoustic absorption coefficient and a compressional sound speed over twice that of soft tissue (see Table 1). This leads to the attenuation and aberration of propagating wavefronts and a reduction in focusing quality $[1,3]$. Acoustic time-reversal (TR) focusing, refined for use in transcranial focusing by Aubry et al [3], takes advantage of the time-invariance of the lossless wave equation, and is able to correct for the aberration of a propagating wavefront. In simulated TR, numerical simulations of the propagation of ultrasound from the target area to an ultrasound transducer are carried out using acoustic property maps of the head, which can be derived from $\mathrm{CT}$ images. The pressures predicted at the simulated transducer are reversed in time and used to generate input phase and amplitude values for a multi-element array source. Marquet et al. [4] demonstrated that simulated TR is
TABLE 1

ACOUSTIC PROPERTIES OF TISSUES IN THE HEAD [9]

\begin{tabular}{llll}
\hline \hline Tissue & $\begin{array}{l}\text { Compressional } \\
\text { sound speed }\left[\mathrm{ms}^{-1}\right]\end{array}$ & $\begin{array}{l}\text { Density } \\
{\left[\mathrm{kgm}^{-3}\right]}\end{array}$ & $\begin{array}{l}\text { Absorption coefficient } \\
{\left[\mathrm{dBcm}^{-1} \mathrm{MHz}^{-1}\right]}\end{array}$ \\
\hline Fat & 1430 & 928 & 0.60 \\
Muscle & 1580 & 1040 & 0.45 \\
Brain & 1560 & 1040 & 0.58 \\
Bone & 3200 & 1990 & 3.5 \\
\hline \hline
\end{tabular}

capable of attaining $90 \%$ of the peak pressure obtained with gold-standard hydrophone based focusing methods when focusing through ex vivo skull bone. Simulated TR can enable millimeter scale focusing with sub-millimeter positioning accuracy, and has since been employed in numerous studies requiring the focused transcranial application of ultrasound. However, simulated TR for transcranial focusing remains subject to error and uncertainty with resultant loss in focusing quality. Four potential sources of error are:

i. The underlying physical model, and how well it accounts for phenomena such as absorption, nonlinearity, and elastic wave propagation.

ii. Errors due to the discretisation of the physical domain, including numerical dispersion, the response to medium heterogeneities, and the effectiveness of the perfectly matched absorbing boundary layer (PML).

iii. The inputs to the model, such as the map of acoustic medium properties and the representation of acoustic transducers.

iv. How numerical simulations are used within a larger TR protocol, including how phenomena that are not timeinvariant, such as absorption, are accounted for.

TR simulations are often carried out using finite difference time-domain (FDTD) schemes, which can be limited in their accuracy and efficiency for large-scale problems. Recently a more advanced k-space corrected pseudospectral time domain (PSTD) scheme was examined for simulated TR and shown to give comparable accuracy with less restrictive sampling criteria [1]. In the present paper, the errors that can arise due to the discretisation of the domain (ii above) are examined in more detail. Their impact on the accuracy of PSTD schemes is determined via numerical testing, and the spatial and temporal sampling criteria required to reduce their impact to an acceptable level are established. 2-2 and 2-4 FDTD schemes are also tested for the purpose of comparison. 


\section{SIMULATION OF ULTRASOUND PROPAGATION}

\section{A. Finite Difference Methods and Numerical Dispersion}

FDTD methods have seen extensive use in simulated TR $[1,3,4]$, but are subject to errors in gradient estimation that result in an unphysical dependence of the sound speed on wave frequency and the spatial and temporal discretisation parameters. This leads to a cumulative error in phase, termed numerical dispersion [5].

PSTD methods replace the finite difference calculation of spatial gradients with a spectral gradient calculation, in this case via a Fourier series. This prevents the numerical dispersion due to spatial discretisation, but not that due to the finite difference approximation of temporal gradients. However, the known value of the dispersion error arising from time stepping can be used to introduce a correction factor, $\kappa=\operatorname{sinc}\left(c_{\mathrm{ref}} k_{x} \Delta t / 2\right)$, in the spatial frequency domain. This is the $\mathrm{k}$-space corrected PSTD method $[6,7]$, and is exactly accurate for homogenous media up to the Nyquist limit. It remains highly accurate in weakly heterogeneous media, but the application of $\kappa$ in the wavenumber domain means that only a single sound speed can be perfectly corrected for. This means that numerical dispersion dependent on temporal sampling will still arise in heterogeneous media. When $c_{\text {ref }}<c$, dispersion is bounded between zero and that of the uncorrected PSTD scheme. For $c_{\text {ref }}>c$ dispersion is not bounded [7]. Formulation of any of these numerical schemes as a system of coupled first-order equations allows implementation of a split-field PML to replicate free field conditions [6]. The PML is intended to absorb incident waves before they reach the edge of the grid without reflecting any pressure from its surface.

\section{B. The Band-Limited Interpolant}

In any collocation method, it is necessary to interpolate between the values at the collocation points. In finite difference methods, polynomials are used to interpolate locally between neighbouring grid points. In PSTD methods, a Fourier series is applied globally to interpolate between the values at all the collocation points simultaneously. Because in practice only a finite number of Fourier components can be included, the representation is inevitably bandlimited, giving rise to Gibbs-type artifacts. This interpolating function, known as the band-limited interpolant (BLI), is shown for a Kronecker delta in Fig. 1 (a). In PSTD methods, it is the gradient of the BLI that is calculated [8]. This is not problematic as long as the BLI is an accurate representation of the variation of the discretely sampled function in between the collocation points.

The BLI will affect attempts to describe discontinuous pressure distributions or medium properties on the grid. The representation of media where there are large step changes in medium properties between grid points has previously been identified as a limitation of PSTD methods $[1,6]$. The magnitude of Gibbs' phenomenon is related to the decay of the Fourier coefficients, and smoothing can be employed to improve the BLI representation, as shown in Fig. 1 (b) for a Blackman window. Although this still does not resemble the (a)

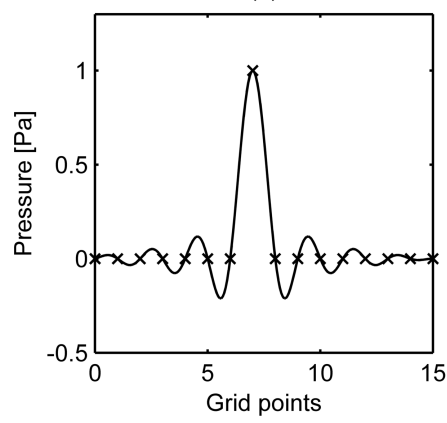

(b)

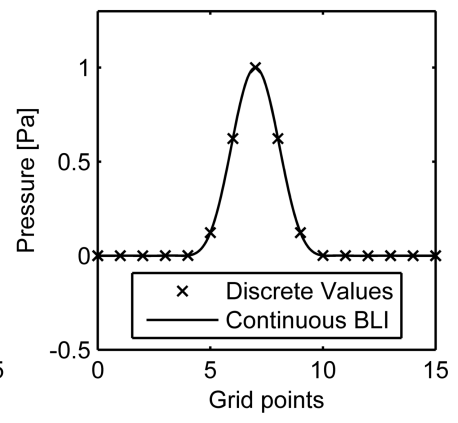

Fig. 1. Spatial delta functions and their band limited interpolants (BLIs). (a) Unsmoothed, and (b) frequency filtered with a Blackman window.

original delta function, the continuous BLI matches the implied underlying pressure distribution more closely.

\section{NUMERICAL TESTS}

\section{A. Overview}

The impact of numerical dispersion, the effectiveness of the PML, and the accuracy of the representation of step-changes in medium properties were examined. Numerical simulation of ultrasound was carried out using the k-Wave toolbox [7]. A fluid model was used; meaning shear wave propagation was not considered. Spatial delta functions filtered with a Blackman window in the spatial frequency domain were used as broadband pressure sources as shown in Fig. 1 (b). Medium properties for both bone and brain tissue were used as defined in Table 1. Tests in 1D were carried out using leapfrog and kspace corrected PSTD schemes, and using staggered-grid 2-2 and 2-4 staggered FDTD schemes for comparison. A 4096point spatial grid was used, with a total length of $0.1 \mathrm{~m}$. Tests in $2 \mathrm{D}$ were carried out using a k-space corrected PSTD scheme on a $256 \times 256$ spatial grid of $0.1 \mathrm{~m} \times 0.1 \mathrm{~m}$. Unless stated otherwise, all simulations used a Courant-FriedrichsLewy number $\left(\mathrm{CFL}=c_{\max } \Delta t / \Delta x\right)$ of 0.3 , and a 20 grid point PML with a peak absorption of 2 Nepers per grid point. Sampling criteria were calculated from acoustic frequency as spatial points per wavelength (PPW $=c / f \Delta x$ ) and temporal points per period $(\mathrm{PPP}=1 / f \Delta t)$. PPW were calculated using the wavelength of sound in brain tissue.

\section{B. Numerical Dispersion}

To examine the impact of numerical dispersion on simulation accuracy, a broadband source was defined as an initial pressure distribution on the grid and the time-varying pressure signal recorded at a distance of $1 \mathrm{~cm}$. PSTD schemes and a 2-4 FDTD scheme were tested. When k-space correction was used, $c_{\text {ref }}$ was set to the sound speed in brain tissue. As the CFL number defines a fixed ratio between spatial and temporal sampling, the test was repeated with the CFL varied from 0.03 to 0.6 to separate dispersion effects due to each discretisation. The phase spectrum of the recorded pulses was computed via fast Fourier transform (FFT) and compared to a reference generated with perfect $\mathrm{k}$-space correction. The error in phase as a function of frequency, $\varnothing(f)$, was converted to a positional error in brain tissue per $\mathrm{cm}$ propagated:

$$
\text { Positional } \operatorname{Error}(f)=c_{\text {brain }} \varnothing(f) / 2 \pi f \text {. }
$$


This was used to calculate the sampling criteria required to reduce the positional error due to numerical dispersion below $1 \mathrm{~mm}$ for a target in the deep brain, assuming $1 \mathrm{~cm}$ propagation through bone and $10 \mathrm{~cm}$ propagation through brain tissue, with an additional $20 \mathrm{~cm}$ propagation through brain tissue per reverberation. Results are shown in Table 2, and demonstrate the increase in accuracy obtained by spectral methods, and then the near-total removal of dispersive error that is attained with $\mathrm{k}$-space correction.

It should be noted that FDTD schemes are dependent on both spatial and temporal sampling making direct comparison with PSTD schemes difficult. For the values in Table 2, the temporal PPP required in the 2-4 FDTD scheme were calculated for a fixed CFL number of 0.3 , meaning that each value corresponds to a distinct spatial sampling rate that also contributes to the dispersive error. To put this in context, with a CFL of 0.03, the FDTD scheme requires 5.2 PPW to obtain $<1 \mathrm{~mm}$ error for the direct path, which corresponds to temporal sampling at $>300$ PPP. Values for the PSTD schemes are valid up to $2 \mathrm{PPW}$.

\section{The Perfectly Matched Layer}

In 1D, frequency filtered delta function initial pressure sources were propagated towards the PML on a homogeneous grid with the acoustical properties of brain tissue. PSTD schemes were tested against a 2-2 FDTD scheme with a CFL of 1 , which prevents numerical dispersion arising. It should be noted that this FDTD formulation is not practical outside the homogenous 1D case due to stability constraints. Time traces of the incident wave and any pressure reflected from the surface of the PML or transmitted to the edge of the grid were recorded, and the amplitude spectra of the recorded traces were obtained via FFT. PML effectiveness as a function of frequency was calculated as the ratio of reflected or transmitted to incident amplitude in decibels. Results are shown in Fig. 2 (a)-(b) for k-space corrected and FDTD schemes only. For both schemes, transmission remains constant at under $-70 \mathrm{~dB}$ until spatial sampling drops beneath 3 PPW, below which the k-space shows varying transmission, and the FDTD scheme shows a reduction in transmission. For both schemes reflection shows a greater dependence on spatial sampling, rising steadily from under $-120 \mathrm{~dB}$ for frequencies sampled at 4 PPW to total reflection at 2 PPW.

The PML was also tested in two dimensions to determine its effectiveness as a function of the angle of incidence of incoming waves. A smoothed point source was placed at the edge of the PML on the 2D grid, and propagated into the PML. The pressure was recorded at the edge of the simulated domain to examine transmission, with each sensor position corresponding to a distinct angle of incidence. The peak

TABLE 2

SAMPLING CRITERIA REQUIRED FOR $<1$ MM DISPERSIVE POSITIONAL ERROR

\begin{tabular}{lllll}
\hline \hline Scheme & $\begin{array}{l}\text { Direct path } \\
{[\text { PPP }]}\end{array}$ & $\begin{array}{l}\text { One Reverb } \\
{[\text { PPP }]}\end{array}$ & $\begin{array}{l}\text { Two Reverbs. } \\
{[\text { PPP }]}\end{array}$ & $\begin{array}{l}\text { Three Reverbs. } \\
{[\text { PPP }]}\end{array}$ \\
\hline FDTD & 34 & 44 & 49 & 53 \\
PSTD & 13 & 23 & 29 & 34 \\
K-space & 4 & 4 & 4 & 4 \\
\hline \hline
\end{tabular}

(a)
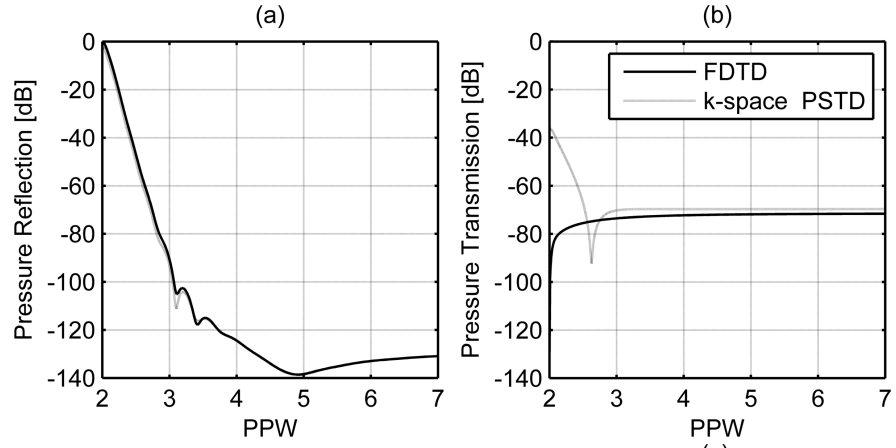

(c)

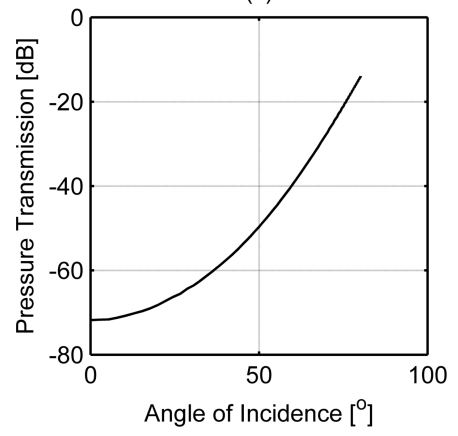

Angle of Incidence $\left[^{\circ}\right]$

Fig. 2. (a) Reflection from and (b) transmission through the $1 D$ PML as a function of spatial points per wavelength (PPW). (c) Transmission to the edge of the grid as a function of angled of incidence.

pressure transmission at each angle was compared with a reference recording obtained with PML absorption set to zero to control for wave spreading, and the ratio calculated in decibels. A clear relationship between the attenuation of the transmitted wave and the angle of incidence was observed as shown in Fig. 2 (c).

\section{Representation of Medium Heterogeneities}

To examine the error originating from the interface between bone and brain-tissue, sources were propagated in both directions across the simulated interface, defined as an unsmoothed step change on the grid. The incident, reflected, and transmitted waves were recorded and their amplitude spectra obtained via FFT. These spectra were then used to calculate simulated pressure reflection and transmission coefficients as a function of frequency. Percentage errors in the simulated coefficients compared to the analytical values were calculated as a function of PPW and are shown in Fig. 3. To obtain $<1 \%$ error in reflection and transmission coefficients, k-space corrected PSTD schemes require 7.5 spatial PPW, while the 2-4 FDTD scheme requires 8.3 PPW.

To examine the effect of medium discontinuities in more detail, this test was repeated with a varying acoustic impedance ratio, ranging from $1: 1$ to $1: 16$. The effect of changes in density and sound speed were examined separately, and the percentage error in pressure transmission and reflection coefficients established in the same manner as with the bone/brain-tissue interface. Fig. 4 shows the percentage error in transmission coefficients as a function of impedance change for a frequency sampled at 10 spatial PPW. It shows that it is the representation of density in the PSTD scheme that causes a reduction in accuracy in highly heterogeneous media. It also shows that the k-space PSTD scheme gives comparable 
(a)

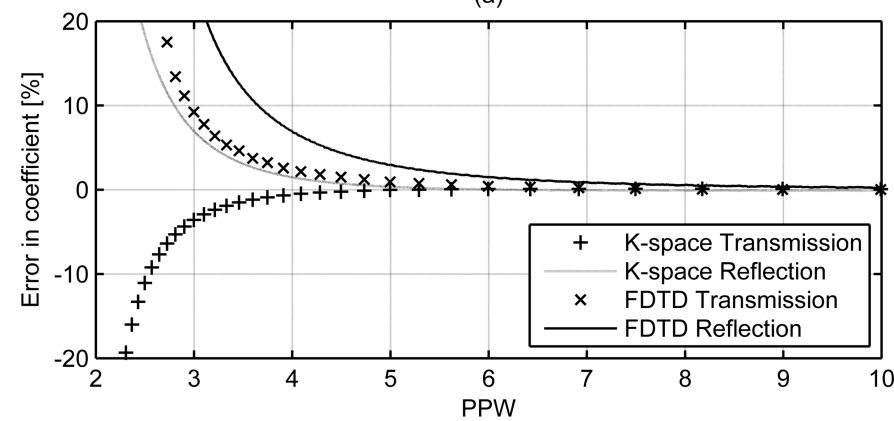

(b)

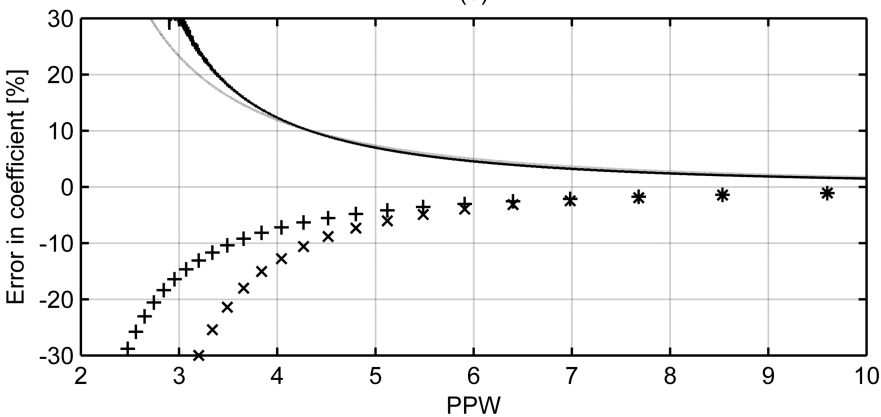

Fig. 3. Percentage error in simulated reflection and transmission coefficients (a) brain-tissue to bone (b) bone to brain-tissue as a function of spatial points per wavelength (PPW).

accuracy to the FDTD scheme until the ratio of the density change at the interface is above $1: 4$, much greater than the difference between bone and soft tissues shown in Table 1 .

\section{CONCLUSIONS}

In this paper the manifestation of errors resulting from the discretisation of the physical domain when performing simulations of transcranial ultrasound propagation with PSTD schemes was examined. Although the effectiveness of PSTD schemes in highly heterogeneous media has previously been identified as a key limitation of such schemes, they were shown to simulate reflection and transmission from changes in medium properties with equal or greater accuracy than equivalent FDTD schemes. Furthermore, Table 2 shows how PSTD schemes can reduce dispersive error with greater efficiency than equivalent FDTD schemes, especially if multiple reverberations within the skull cavity need to be simulated. Combined, these results recommend PSTD methods with k-space correction for use in TR for transcranial focusing. A dependence of PML effectiveness on spatial sampling was observed for both schemes, as well as a dependence on angle of incidence, but it is expected that the spatial sampling required to control other sources of error, combined with sensible simulation geometry, should prevent the PML from becoming ineffective.

It should be noted that this is not an exhaustive description of the impacts of these sources of error, and these tests are subject to some limitations. For instance, when reflection and transmission from medium step changes were tested, PSTD and FDTD schemes both remained dispersive. Care was taken to ensure that distinct incident, reflected, and transmitted waves were recorded, but it is possible this may have affected results. Furthermore, when testing the PML in 2D, a straightline path from the initial source to each sensor was assumed,

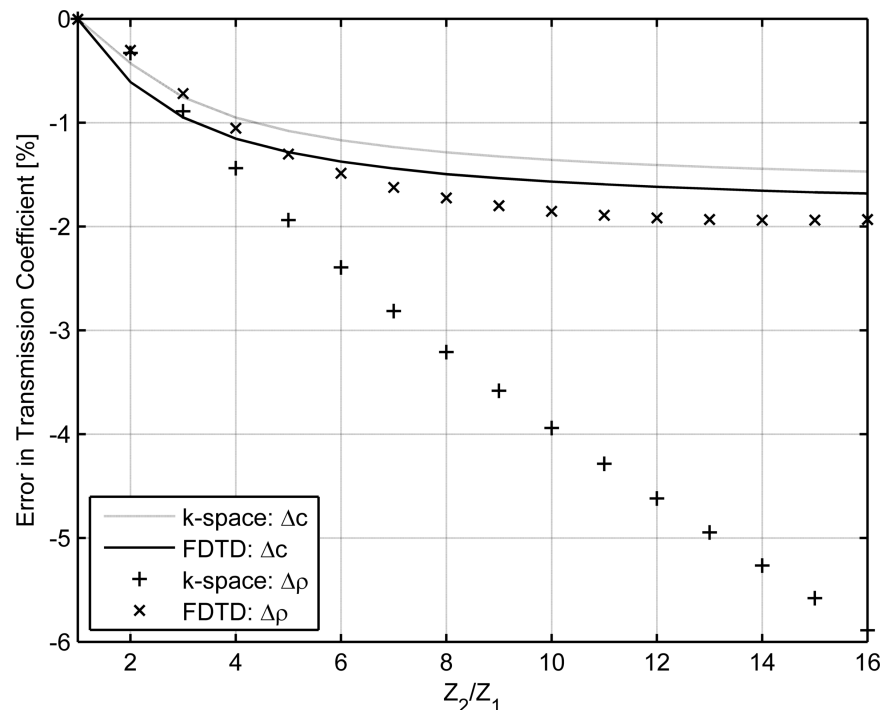

Fig. 4. Error in simulated transmission coefficient of a frequency sampled at 10 points per wavelength, as a function of impedance change.

enabling calculation of angle of incidence. It is possible that refraction of the wavefront by the absorbing PML may invalidate this assumption, although this was not observed. Finally, an additional discretisation error, staircasing, was not examined here. Staircasing refers to the stair-stepped representation of geometries on a discrete Cartesian grid in 2D and $3 \mathrm{D}$, which can affect the source, sensor, and medium representation on the grid. The impact of staircasing is expected to depend on the particular medium geometry, and also to be reduced by finer spatial discretisation.

As discussed in Section I, errors due to the discretisation of the physical domain are one of several potential sources of error in simulated TR. Future work should make use of the guidelines for effective sampling criteria established here to examine the effects of staircasing and the tolerance of simulated TR protocols to the additional uncertainties discussed in Section I, with the aim of improving the efficiency and focusing effectiveness of the method.

\section{REFERENCES}

[1] Y. Jing, F. C. Meral, and G. T. Clement, "Time-reversal transcranial ultrasound beam focusing using a k-space method.," Phys. Med. Biol., vol. 57, no. 4, pp. 901-17, 2012.

[2] W. Legon et al., "Transcranial focused ultrasound modulates the activity of primary somatosensory cortex in humans.," Nat. Neurosci., pp. 322-329, 2014.

[3] J. F. Aubry et al "Experimental demonstration of noninvasive transskull adaptive focusing based on prior computed tomography scans.," J. Acoust. Soc. Am., vol. 113, pp. 84-93, 2003.

[4] F. Marquet et al., "Non-invasive transcranial ultrasound therapy based on a 3D CT scan: protocol validation and in vitro results.," Phys. Med. Biol., vol. 54, no. 9, pp. 2597-613, 2009.

[5] J. W. Thomas, Numerical Partial Differential Equations: Finite Difference Methods. New York, USA: SPRINGER, 1995.

[6] M. Tabei, T. D. Mast, and R. C. Waag, "A k-space method for coupled first-order acoustic propagation equations.," J. Acoust. Soc. Am., vol. 111, no. 1 Pt 1, pp. 53-63, 2002.

[7] B. E. Treeby, J. Jaros, A. P. Rendell, and B. T. Cox, "Modeling nonlinear ultrasound propagation in heterogeneous media with power law absorption using a k-space pseudospectral method.," $J$. Acoust. Soc. Am., vol. 131, no. 6, pp. 4324-36, 2012.

[8] L. N. Trefethen, "Spectral Methods in Matlab," Lloydia Cincinnati, vol. 10,2000

[9] F. Duck, "Physical Properties of Tissue. A Comprehensive Reference Book," Medical Physics, vol. 18. Academic Press, London, p. 834, 1991. 